\title{
Perceptions of patients with rheumatoid arthritis about self-assessment of disease activity after watching an educational video: a qualitative pilot study from the AUTO-DAS in Middle Eastern Arab countries project
}

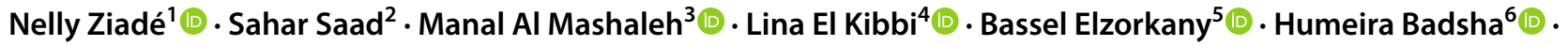

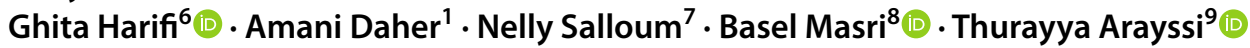

Received: 17 December 2020 / Accepted: 21 January 2021 / Published online: 6 February 2021

(C) The Author(s), under exclusive licence to Springer-Verlag GmbH, DE part of Springer Nature 2021

\begin{abstract}
To evaluate the perceptions of patients with rheumatoid arthritis (RA) about self-assessment of their disease activity after watching an educational video. Consecutive patients with RA consulting their rheumatologist in six Middle Eastern Countries were invited to watch an educational video developed to teach self-assessment using Disease Activity Score (DAS-28). Then, a rheumatology nurse conducted a semi-structured interview and collected the patients' perception about the understanding of the video, feasibility, capability and confidence in performing self-assessment using Likert-type items. The degree of confidence with self-assessment was correlated to the patients' socio-demographic characteristics. Sixty-two patients were included and had an overall positive reaction to the video. It was easy to understand in $96 \%$ and helped facilitate selfassessment in $92 \%$ of cases. Self-assessment was considered totally feasible in $74 \%$, and $66 \%$ of patients were capable of always doing it, with a confidence of $60 \%$ (always) to $34 \%$ (sometimes). Confidence was associated with a higher educational level. Nevertheless, $77 \%$ of patients felt that the self-assessment would not fully replace the physician's visit. Open-ended questions identified five themes: better understanding of the disease, easier communication with the rheumatologist, less consultation time, difficulty with the scoring part and importance of practice. Patients with RA felt that self-assessment was feasible and helpful in understanding RA, improving communication with the rheumatologist and shortening the visit time.
\end{abstract}

Keywords Patient education $\cdot$ Patient empowerment $\cdot$ Disease activity $\cdot$ Educational video $\cdot$ Rheumatoid arthritis $\cdot$ Social distancing

The study was presented as a poster at the 2018 ACR/ARHP Annual Meeting and published in the form of an abstract as: Ziade N, Saad S, al Mashaleh M, el Kibbi L, el Zorkany B, Badsha H, Harifi G, Daher A, Salloum N, Masri B, Arayssi T. Perceptions of Patients with Rheumatoid Arthritis about Self-Assessment of Disease Activity after Watching an Educational Video: Qualitative Pilot Results from the Auto-DAS in Middle Eastern Arab Countries Study [abstract]. Arthritis Rheumatol. 2018; 70 (suppl 10). https://acrabstracts.org/abstract/perceptions-of-patientswith-rheumatoid-arthritis-about-self-assessment-of-disease-activ ity-after-watching-an-educational-video-qualitative-pilot-resul ts-from-the-auto-das-in-middle-eastern-arab-coun/.

Nelly Ziadé

nellziade@yahoo.fr; nelly.zoghbi@usj.edu.lb

Extended author information available on the last page of the article

\section{Introduction}

Treating rheumatoid arthritis (RA) to target and using composite measures to assess disease activity regularly is the current standard of care in rheumatology $[1,2]$ and has shown a clear benefit in improving the disease prognosis over the past decade [3-5].

Moreover, empowering patients through education and involving them in disease management through a shared decision process are recommended by major rheumatology associations [6, 7]. Those strategies improve patients' perception of disease activity in-between visits and enhance treatment adherence. They also allow the rheumatologist to make better informed therapeutic decisions while saving precious time at the consultation. 
Many tools for measuring disease activity, such as the Disease Activity Score (DAS-28), are now accessible to patients in various formats such as e-health platforms, web applications, and smartwatches [8-13]. Moreover, using videos to educate patients about using these tools has several advantages over brochures and written text. Education through videos has shown efficacy in modifying patient behavior, mainly when using a standardized patient enacting this behavior. It is also an effective method to convey complicated medical concepts in a simple and accessible manner to a layperson [14]. Furthermore, it is time-efficient, round-the-clock accessible, and allows the learners to proceed at their own pace [15]. It may be of particular interest in times where social distancing is recommended, such as the COVID-19 pandemic.

Our study team developed and validated an educational video in Arabic to teach patients with RA self-assessment of their disease activity using DAS-28 (termed auto-DAS) [16]. The video was based on two previously published videos, both tested with real-life patients, in English [17, 18] and French $[19,20]$. It resulted from the collaboration of a team of twenty-three rheumatology experts from the Middle Eastern Arab Countries (MEAC) (study team) with a patient with RA, a medical student, a rheumatology nurse, and a professional technical team. The video featured a real patient as a model and was culturally adapted to the MEAC. It had a seven minutes duration, was easily transferable on the patient's smartphone, and usable without the need for an internet connection [21].

The primary objective of this study was to evaluate the perceptions of patients with RA about self-assessment of their disease activity using DAS-28 after watching the educational video. The secondary objectives were to collect the patient's feedback about understanding the video and to identify patients' characteristics associated with their confidence in self-assessment.

\section{Methods}

\section{Study material}

The video was created for Arab-speaking patients with RA and was previously validated by the study team and a sample of patients with RA for content [16, 22]. It consisted of a short introductory note about the assessment of disease activity in RA, followed by a demonstration of the evaluation of each of the 28 joints for pain and swelling, performed by a real patient with RA, and finally an explanation about the final score calculation and the categorization into the disease activity levels.

The questionnaire inquiring about the patients' perceptions about self-assessment after watching the video was designed by 9 rheumatologists among the authors, based on their clinical practice. Validation of the content was made by a panel of 23 rheumatologists from the Arab countries through e-mail discussions. In addition, validation of the format was made by a study nurse and a medical student to ensure that the language was clear and that the questions were not ambiguous [23]. The final semi-structured interview consisted of a unified pre-defined scenario comprising a combination of qualitative open-ended items and Likerttype items with responses from 1 (strongly disagree) to 3 (strongly agree) (Supplementary Table 1).

\section{Patient recruitment}

We invited consecutive adult patients with RA in six MEAC (Bahrain, Egypt, Jordan, Lebanon, Saudi Arabia and the United Arab Emirates (UAE)) to participate in the study during a routine rheumatology visit. The patients who did not understand the Arabic language or could not read the English subtitles were excluded from the study. In addition, patients with a newly made diagnosis (less than 3 months before the study inclusion) were excluded.

\section{Study procedure}

During the routine clinic visit, the rheumatologist explained to the patients the purpose of the study. The patients were then interviewed by the rheumatology nurse and watched the video together on an electronic tablet just before starting the interview. The patients were first asked if the video was easy to understand (binary response yes/no). Then, the semi-structured interview was conducted with the nurse. The interviewer reassured the patient that the data will be entirely anonymized and that their identity will not be revealed during the analysis and the publication of the results. The data was recorded on paper then transferred to an Excel sheet.

In parallel, the demographic and disease data were collected, including date of birth, gender, marital status (married/other), educational level (illiterate/elementary school/ high school/university), profession (white collar/blue collar/ retired/at home), weight, height, duration of RA symptoms, date of RA diagnosis, seropositivity, radiographic damage, current RA treatment (conventional synthetic DiseaseModifying Anti-Rheumatic Drugs (DMARDs)/biologic DMARDs, targeted synthetic DMARDs/corticosteroids), and general comorbidities (smoking, hypertension, osteoporosis, dyslipidemia, diabetes, and fibromyalgia).

\section{Statistical analysis}

Four authors (NZ, LK, NS, TA) reviewed the transcribed responses to the open-ended questions, analyzed the 
responses and classified them into homogeneous themes. Any discrepancies were resolved by discussion.

The demographic and clinical data and the Likert-type items were presented descriptively. The question about the confidence in self-assessment was transformed in binary responses ("always" versus "sometimes" + "never") and correlated with the patients' and disease's characteristics (bivariate analysis using chi-square or Fisher's test for categorical variables, and $T$ test for numerical variables; multivariable analysis using binary logistic regression). The statistical analysis was conducted using SPSS V22.0.

\section{Sample size calculation}

Since the study is qualitative, there's no consensus in the literature about the ideal sample size. Some authors recommend including 12-30 patients if the patients are homogeneous [24, 25]. Since we included patients from different Arab countries, particularly from three global regions (Levant, Gulf and North Africa), we multiplied the above approximative number by three and reached 62 patients.

\section{Ethical considerations}

The study was approved by the Central Ethics Committee (Saint-Joseph University, Beirut (Tfem 2018/24) and by the Ethics Committees of all the participating centers. All the patients signed an informed consent form.

\section{Results}

All the patients who were invited to participate in the study agreed to be interviewed. Sixty-two patients were included (20 from Lebanon, 13 from Bahrain, 10 from Saudi Arabia, 9 from Jordan, 6 from Egypt and 4 from the UAE). Their mean age (SD) was 51.2 years (13.4), 86\% were female, and $40 \%$ had a university education (Table 1 ). The mean disease duration (SD) was 8.4 years (6.4), and the average time since diagnosis was 1.9 years (6.4). The interview lasted around $15 \mathrm{~min}$, in addition to the $7 \mathrm{~min}$ duration of the video.

Overall, the reaction to the video was positive (Table 2). The video was found easy to understand in $96 \%$ of cases. In addition, 92\% stated that the video facilitated selfassessment. Performing their self-assessment was found totally feasible in $74 \%$, and partially feasible in $23 \%$ of the patients. Ninety-five percent of the patients felt they were totally (66\%) or partially (29\%) capable of doing their self-assessment.

The degree of confidence in performing self-assessment was $60 \%$ (always confident) and 34\% (sometimes confident). Almost half of the patients (47\%) said that they had higher confidence in performing self-assessment during a disease
Table 1 Patients characteristics

\begin{tabular}{ll}
\hline Patients' characteristics & 62 patients \\
\hline Mean age & 51.2 yo (SD 13.4) \\
Female \% & $86 \%$ \\
University education \% & $40 \%$ \\
Rheumatoid arthritis mean duration & 8.4 years (SD 6.4) \\
Average time to diagnosis & 1.9 years (SD 6.4) \\
Positive rheumatoid factor & $67 \%$ \\
Positive anti-citrullinated proteins antibodies & $46 \%$ \\
Bone erosions & $48 \%$ \\
Biologic treatment & $32 \%$ \\
Targeted synthetic disease-modifying anti-rheu- & $11 \%$ \\
matic drugs & \\
Corticosteroids & $24 \%$ \\
Common comorbidities & \\
Smoking & $19 \%$ \\
Hypertension & $23 \%$ \\
Osteoporosis & $13 \%$ \\
Dyslipidemia & $11 \%$ \\
Diabetes & $11 \%$ \\
Fibromyalgia & $6 \%$ \\
\hline
\end{tabular}

flare, rather than during a remission phase (15\%). In this regard, they reported that, during a disease flare, they would be absolutely sure that the joint count is correct, regarding both tender and swollen joints. However, during remission, they would not be certain if the joints were absolutely clear of swelling, and they were afraid of underestimating the swollen joint count.

Around half of the patients (45\%) said they would always continue to do their self-assessment in the long term, and a third $(31 \%)$ stated that they would perform it some of the time.

Finally, there was a clear trend in expressing that selfassessment would not replace the physician's visit, whether fully $(77 \%)$ or partially (18\%).

The confidence in performing self-assessment was associated with marital status (married patients felt more confident, $p=0.018$ ), education (university level patients felt more confident, $p=0.024$ ), and corticosteroid therapy (patient without corticosteroid therapy felt more confident, $p=0.005)$ in bivariate analysis. Only education remained associated in multivariable analysis $(p<0.001)$.

The analysis of the open-ended questions identified five general themes (Fig. 1), of whom three were positive perceptions: watching the video and the idea of self-assessment were associated with a better understanding of the disease (education), an easier communication with the rheumatologist, and less consultation time. The fourth theme was expressing the difficulty with the calculation segment of the video, as the patients found that the scoring part of 
Table 2 RA patients' responses to the closed questions of the interview

Answers $N(\%)$

How feasible is it for you to do your own assessment of your disease activ- $\quad$ Feasible 46 (74) Partially feasible 14 (23) Not feasible 1 (2) ity using auto-DAS?

Do you feel capable of doing your own assessment using auto-DAS?

Do you feel confident in doing your own assessment using auto-DAS?

When would you feel more confident in doing your own assessment using auto-DAS?

Will you continue to do your own self-assessment using auto-DAS in the long term?

Do you think that your own evaluation of your disease using auto-DAS can Yes 1 (2)

Always 41 (66) Partially 18 (29)

Always 37 (60) Sometimes 21 (34)

Remission 9 (15) Flare 29 (47)

Always 28 (45) Sometimes 19 (31)

Partially 11 (18)

Partially 3 (5)

Do you think that the video was helpful in facilitating your self-assessment Yes 57 (92) using auto-DAS?

\section{Never 2 (3)}

Never 3 (5)

No difference 23 (37)

I do not know 11 (18)

No 48 (77)

No $0(0)$
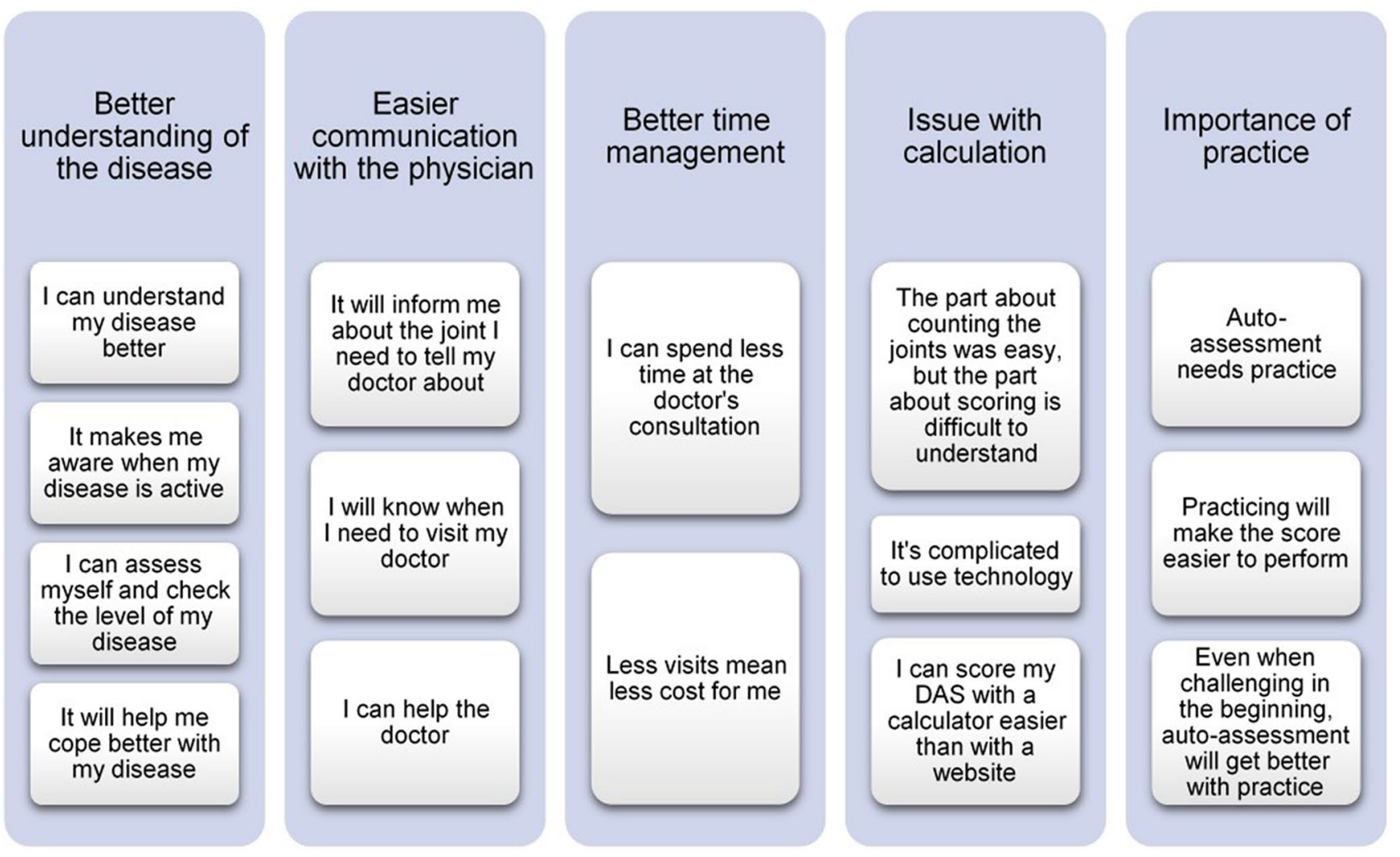

Fig. 1 Thematization of the responses to the open-end questions, with illustrative quotes

auto-DAS was challenging. However, the fifth theme was the importance of practice to overcome the scoring challenge. The themes are presented hereafter with some illustrative quotes. The number of times a quote is mentioned is in parenthesis:

- Better understanding of the disease (education): "I can understand my disease better" (9), "I can assess myself and check the level of my disease" (8), "it will make me aware when my RA is active" (3), "it is particularly useful to new patients at the beginning of the disease" (2), "it gives me a good experience" (1), "I can understand my symptoms better and cope better with my disease" (1)

- Easier communication with the physicians: "I will know when I need to visit my doctor" (4), "it will inform me about the joint I need to tell my doctor about" (3), "I can help the doctor" (1). 
- Better time management (less consultation time): "I can spend less time at the doctor's consultation" (7), "less visit" (2), "less visits mean less cost for me" (1).

- Difficulty with the scoring: "the part about counting the joints was easy, but the part about scoring is difficult to understand" (1), "I found it hard to calculate the autoDAS" (1), "I can score my DAS with a calculator easier than with a website" (1), "I'm not sure I can do it" (1), "I feel it's difficult" (1), "I believe that only the doctor can assess my RA" (1), "It's complicated to use technology" (1), "my wife can help me" (1).

- Importance of practice: "self-assessment needs practice" (34), "I need to gain experience" (3), "I need help" (1), "practicing will make the score easier to perform" (1), "even when challenging in the beginning, self-assessment will get better with practice" (1), "It's difficult the first time you see it, then it's easy" (1), "It's only difficult at the beginning" (1).

The most difficult parts to understand were: data entry and calculation (6\%), examining the knees (3\%), examining the hands (3\%), and checking the swelling in the fingers $(2 \%)$.

\section{Discussion}

Overall, the perceptions of the patients with RA about selfassessment of their disease activity, based on watching the educational video about DAS-28, were positive.

The video was easy to understand by most of the patients and was found helpful in facilitating their self-assessment. Around two-thirds of the patients felt capable of performing their self-assessment and were confident in their performance. Married patients felt more confident, which may be due to counting on their partner's support in helping them in self-assessment. In addition, higher education was associated with higher confidence, which is an expected finding [26]. Finally, the absence of corticosteroid therapy was associated with higher capability in self-assessment. This may reflect a category of patients with less severe RA, thus a lower need to be examined by the rheumatologist.

Compared to the available English and French videos, our video was shorter (seven minutes compared to around twenty minutes in the other videos), making it easier to watch in full. It was also available on the patient's smartphone and could be viewed without an internet connection, which facilitates access at any time. Our patients' perceptions about the auto-DAS video were similar to the evaluation of the English and French videos by patients with RA [18-20]. In fact, both evaluations showed that training patients to carry out their self-assessment with DAS-28 was feasible and acceptable and that most patients adhered to reporting their auto-DAS as per an evaluation after six months in the English study. However, in the French study, whose primary endpoint was measured at three years, adherence to self-assessment decreased over time, indicating a necessity to reinforce the communication about auto-DAS. On the other hand, the English study patients felt that their new ability to use the DAS-28 improved their understanding of the disease in $65 \%$ of the cases. In addition, the latter study demonstrated better performance with time, as the correlation between the DAS28 performed by the patients and by a nurse specialist tended to become more and more similar after the study period of six months. This supports our patient's statement that their assessment would become better with time and practice. Around half of the patients stated that they would continue to do their self-assessment at home, which is in concordance with the French study, which stated that 55\% of the French patients would carry on their self-assessment regularly [19].

As in other educational videos, whether in rheumatology [18-20] or other fields, such as vaccination [27] or cancer health literacy [28], the patients really appreciated the education that is offered to them.

The study identified the areas of difficulty in the video. Although the joint count segment seemed feasible, using an online calculator to compute the final score was challenging. However, most of the patients felt that practicing self-assessment will make the calculation easier to perform.

By encouraging self-assessment of disease activity, some physicians may be concerned about a loss of income created by a decrease in patients' visits, as they may feel confident that their disease is under control and thus cancel their appointments. However, there was a clear report by our patients that the self-assessment will not totally replace the physician's visit. Moreover, self-assessment of disease activity, when used with the right patients, is likely to improve disease outcomes and patients' adherence to the management plan [29-31]. Furthermore, in the setting of a busy practice, the precious time saved from shortened or fewer follow-up visits can provide additional consultation time slots to offer care for more patients in more need to see the rheumatologist, potentially increasing both income and patient satisfaction.

The study has some limitations. Forty percent of our patients had a university-level education, which may not represent all practices across the Arab world, thus limiting the external validity of our results. In addition, some patients found difficulty in self-assessment, hence they would be reluctant to remain performing auto-DAS. We believe that education about self-assessment is not intended for all patients and might be offered to some patients' categories, such as those with higher levels of education or those who have support at home. 
Nevertheless, the study also has major strengths. First, the response rate was high, which can be explained by the invitation coming directly from the rheumatologist during a routine visit. Second, our study was multicenter, covering six MEAC. Third, it identified the areas of difficulty in selfassessment, which may help to emphasize these areas when performing patient education. Fourth, the strengths of the self-assessment through the educational video, as identified by the patients, were a better understanding of the disease, better coping with RA, time-saving, and better communication with the physician. In fact, the Treat to Target recommendations stated that a shared decision process would enable the patient to help the physician making better informed decisions about disease management [1]. Finally, our study provides an essential tool for the application of telemedicine. This is particularly important for countries with low health budgets, for patients who live in remote areas and during periods where social distancing is recommended, such as in the COVID-19 pandemic.

\section{Conclusion}

The majority of patients with RA felt that self-assessment, based on the educational video, was feasible and helpful in understanding their disease, improving communication with the rheumatologist and shortening the visit time. Most patients stated that it will not replace the physician's visit but will help improve coping with the disease and informing the physician before making therapeutic decisions. Our findings need to be evaluated in a longitudinal prospective study, to assess adherence to self-assessment and impact on disease prognosis.

Supplementary Information The online version contains supplementary material available at https://doi.org/10.1007/s00296-021-04799-2.

Acknowledgements The authors would like to acknowledge the patients for participating in the study and the assistants/ students/ nurses who assisted in the data collection: Dr. Fatima Abdul Majeed Al Hawaj, M. Atef Ahmed, M. Mohammad Alhusamiah, Ms Raquel De Guzman, Ms Lina Razzouk.

Author contributions NZ and TA designed the study. NZ, SS, MM, LK, BZ, HB and GH included patients. NZ, LK, NS, TA reviewed the transcribed responses to the open-ended questions, analyzed the responses and classified them into homogeneous themes. NS conducted semi-structured interviews. AD performed data entry. NZ performed the statistical analysis and drafted the manuscript. All the authors made substantial contributions to the conception and design of the work, to the interpretation of data for the work, to the manuscript drafting and revising revised the manuscript critically for important intellectual content. All the authors approved the final version to be published and agree to be accountable for all aspects of the work in ensuring that questions related to the accuracy or integrity of any part of the work are appropriately investigated and resolved.
Funding None.

Data availability Data can be made available by contact with the corresponding author.

\section{Compliance with ethical standards}

Conflict of interest The authors declare no conflict of interest related to this study, including connections to the pharmaceutical agencies. Connections with pharmaceutical companies NOT related to this study: Nelly Ziade: Speaker's honoraria (Roche, Abbvie, Eli Lilly, Pfizer, Janssen, Novartis, Pierre Fabre, Apotex, Pharmaline), Internal workshops (Abbvie, Eli Lilly, Sanofi-Aventis, Pfizer, Janssen), Advisory boards (Roche, Abbvie, Eli Lilly, Pfizer, Janssen, Novartis, Gilead, NewBridge), Research grants (Abbvie, NewBridge, Algorithm/ Celgene). Sahar Saad: none declared. Manal Al Mashaleh: none declared. Lina el-Kibbi: Research grant (Lilly). Bassel el-Zorkany: Consultancy, Research Grants and Advisory and speakers' honoraria for: Abbott, Abbvie, Amgen, Aspire, BMS, Hekma, Janssen, Lilly, MSD, New Bridge, Novartis, Pfizer, Roche, Sanofi-Aventis, Servier, UCB. Humeira Badsha: Speaker's honoraria (Lilly, Abbvie, Pfizer, Janssen, Novartis Gilead), Advisory board meetings (Lilly, Abbvie, Novartis, Pfizer Gilead); Research grants (Pfizer and Lilly). Ghita Harifi: Speaker fees (Lilly, Abbvie, Pfizer, Janssen, Novartis), Advisory board meetings (Lilly, Abbvie, Novartis, Pfizer), Research grants (Pfizer, Lilly). Amani Daher: non declared. Nelly Salloum: none declared. Basel Masri: Speaker's honoraria (Amgen, Novo Nordisk, Novartis), Advisory board (Amgen). Thurayya Arayssi: none declared.

Ethics approval The study complies with the Declaration of Helsinki. The Ethics Committee of Saint-Joseph University, Beirut has approved the research protocol (Tfem2018/24).

Consent to participate Written informed consent has been obtained from the subjects prior to inclusion in the study.

Consent for publication All the authors have given consent for publication.

\section{References}

1. Smolen JS, Breedveld FC, Burmester GR et al (2015) Treating rheumatoid arthritis to target: 2014 update of the recommendations of an international task force. Ann Rheum Dis 75:3-15. https ://doi.org/10.1136/annrheumdis-2015-207524

2. Smolen JS, Aletaha D, Bijlsma JWJ et al (2010) Treating rheumatoid arthritis to target: recommendations of an international task force. Ann Rheum Dis 69:631-637. https://doi.org/10.1136/ ard.2009.123919

3. Stoffer MA, Schoels MM, Smolen JS et al (2016) Evidence for treating rheumatoid arthritis to target: results of a systematic literature search update. Ann Rheum Dis 75:16-22. https://doi. org/10.1136/annrheumdis-2015-207526

4. Jurgens MS, Welsing PMJ, Jacobs JWG (2012) Overview and analysis of treat-to-target trials in rheumatoid arthritis reporting on remission. Clin Exp Rheumatol 30:S56-63 (PMID: 23078808)

5. Scott IC, Ibrahim F, Panayi G et al (2019) The frequency of remission and low disease activity in patients with rheumatoid arthritis, and their ability to identify people with low disability and normal quality of life. Semin Arthritis Rheum 49:20-26. https://doi. org/10.1016/j.semarthrit.2018.12.006 
6. Zangi HA, Ndosi M, Adams J et al (2015) EULAR recommendations for patient education for people with inflammatory arthritis. Ann Rheum Dis 74:954-962. https://doi.org/10.1136/annrheumdi s-2014-206807

7. Singh JA, Saag KG, Bridges SL et al (2016) 2015 American college of rheumatology guideline for the treatment of rheumatoid arthritis. Arthritis Care Res (Hoboken) 68:1-25. https://doi. org/10.1002/acr.22783

8. McDougall JA, Ferucci ED, Glover J, Fraenkel L (2017) Telerheumatology: a systematic review. Arthritis Care Res (Hoboken) 69:1546-1557. https://doi.org/10.1002/acr.23153

9. Grainger R, Townsley H, White B et al (2017) Apps for people with rheumatoid arthritis to monitor their disease activity: a review of apps for best practice and quality. JMIR mHealth uHealth 5:e7. https://doi.org/10.2196/mhealth.6956

10. Seppen BF, L'ami MJ, Duarte dos Santos Rico S et al (2020) A smartphone app for self-monitoring of rheumatoid arthritis disease activity to assist patient-initiated care: protocol for a randomized controlled trial. JMIR Res Protoc 9:e15105. https://doi. org/10.2196/15105

11. Mcbain H, Shipley M, Olaleye A et al (2016) A patient-initiated DMARD self-monitoring service for people with rheumatoid or psoriatic arthritis on methotrexate: a randomised controlled trial. Ann Rheum Dis 75:1343-1349. https://doi.org/10.1136/annrh eumdis-2015-207768

12. Van Riel P, Alten R, Combe B et al (2016) Improving inflammatory arthritis management through tighter monitoring of patients and the use of innovative electronic tools. RMD Open 2:1-9. https ://doi.org/10.1136/rmdopen-2016-000302

13. Gossec L, Cantagrel A, Soubrier M et al (2019) Sanoïa, plateforme e-santé interactive d' auto-évaluation dans la polyarthrite rhumatoïde. Essai comparatif randomisé de 12 mois sur. Rev Rhum 86:52-58. https://doi.org/10.1016/j.rhum.2018.08.002

14. Abu Abed M, Himmel W, Vormfelde S, Koschack J (2014) Video-assisted patient education to modify behavior: a systematic review. Patient Educ Couns 97:16-22. https://doi.org/10.1016/j. pec.2014.06.015

15. The Ohio state university medical (2006) Effective use of audiovisual materials. https://ogg.osu.edu/media/documents/health_lit/ effective_av.pdf. Accessed 17 Jan 2021

16. Ziade N, Karam GA, Daher A et al (2018) Development ans implementation of an educational video that instructs patients with rheumatoid arthritis for self-assessment of disease activity: methodology of the auto-das in middle eastern arab countries study. Ann Rheum Dis 77:561-562

17. Know your DAS: national rheumatoid arthritis society. https:// nras.org.uk/resource/know-your-das/. Accessed 17 Jan 2021

18. Shaffu S, Edwards J, Neame R, Hassan W (2013) Self-assessment of 28-joint disease activity scores by patients with rheumatoid arthritis on anti-TNF therapy. Rheumatol (UK) 52:576-578. https ://doi.org/10.1093/rheumatology/kes383

19. Dougados M, Soubrier M, Perrodeau E et al (2015) Impact of a nurse-led programme on comorbidity management and impact of a patient self-assessment of disease activity on the management of rheumatoid arthritis: Results of a prospective, multicentre, randomised, controlled trial (COMEDRA). Ann Rheum Dis 74:1725-1733. https://doi.org/10.1136/annrheumdis-2013-20473 3
20. Gossec L, Fayet F, Soubrier M et al (2019) Is self-assessment by patients of disease activity acceptable over the long term in rheumatoid arthritis? A 3-year follow-up of 771 patients. Rheumatol (United Kingdom) 58:1498-1499. https://doi.org/10.1093/rheum atology/kez094

21. AUTOAS-MEAC (2018) AUTODAS MEAC. In: YouTube. https $: / / w w w . y o u t u b e . c o m / w a t c h ? v=o p 23 r 8 d f 9 d Q \& t=364 s$. Accessed 17 Jan 2021

22. Ziade N, Arayssi T, El-Zorkany B, Daher A, Abi Karam G, Abu Jbara M, Aiko A, Alam E, Al Emadi S, Al Mashaleh M, Badsha B, El Kibbi L, Halabi H, Harifi G, Khan B, Masri A MB (2020) Development of an educational video for self-assessment of patients with RA: steps, challenges, and responses. Mediterr J Rheumatol (accepted)

23. Gaur PS, Zimba O, Agarwal V, Gupta L (2020) Reporting survey based studies: a primer for authors. J Korean Med Sci 35:1-15. https://doi.org/10.3346/jkms.2020.35.e398

24. Moser A, Korstjens I (2018) Series: practical guidance to qualitative research. Part 3: sampling, data collection and analysis. Eur J Gen Pract 24:9-18. https://doi.org/10.1080/13814788.2017.13750 91

25. Trotter RT (2012) Qualitative research sample design and sample size: Resolving and unresolved issues and inferential imperatives. Prev Med (Baltim) 55:398-400. https://doi.org/10.1016/j.ypmed .2012.07.003

26. Jiang X, Sandberg MEC, Saevarsdottir S et al (2015) Higher education is associated with a better rheumatoid arthritis outcome concerning for pain and function but not disease activity: results from the EIRA cohort and Swedish rheumatology register. Arthritis Res Ther 17:1-9. https://doi.org/10.1186/s13075-015-0836-6

27. Brown T, Goldman S, Persell S, Lee J, Doan C, Stephens Q, Baker KC (2017) Development and evaluation of a patient education video promoting pneumococcal vaccination. Patient Educ Couns 100:1024-1027

28. Montealegre J, Gossey J, Anderson M, Chenier R, Chauca G, Rustveld MJ-W (2014) Implementing targetes cervical cancer screening videos at the point of care. Patient Educ Couns 97:426-429

29. Goekoop-Ruiterman YPM, De Vries-Bouwstra JK, Kerstens PJSM et al (2010) DAS-driven therapy versus routine care in patients with recent-onset active rheumatoid arthritis. Ann Rheum Dis 69:65-69. https://doi.org/10.1136/ard.2008.097683

30. Barrie J (2011) Patient empowerment and choice in chronic pain management. Nurs Stand 25:38-41. https://doi.org/10.7748/ns201 1.04.25.31.38.c8432

31. Nadrian H, Morowatisharifabad MA, Bahmanpour K (2011) Development of a rheumatoid arthritis education program using the PRECEDE_PROCEED model. Heal Promot Perspect 1:11811829. https://doi.org/10.5681/hpp.2011.013

Publisher's Note Springer Nature remains neutral with regard to jurisdictional claims in published maps and institutional affiliations. 


\section{Authors and Affiliations}

\section{Nelly Ziadé ${ }^{1}$ (D) Sahar Saad ${ }^{2} \cdot$ Manal Al Mashaleh $^{3}$ (D) - Lina El Kibbi ${ }^{4}$ (D) Bassel Elzorkany ${ }^{5}$ (D) Humeira Badsha BD $^{6}$. Ghita Harifi $^{6}$ (D) Amani Daher ${ }^{1} \cdot$ Nelly Salloum ${ }^{7} \cdot$ Basel Masri $^{8} \mathbb{D} \cdot$ Thurayya Arayssi $^{9}$ (D)}

Sahar Saad

saharsaad68@gmail.com

Manal Al Mashaleh

manal_mashaleh@yahoo.com

Lina El Kibbi

linakibbe@hotmail.com

Bassel Elzorkany

basselelzorkany@gmail.com

Humeira Badsha

doctorbadsha@gmail.com

Ghita Harifi

drharifi@drbadshamedical.com

Amani Daher

amydaher25@gmail.com

Nelly Salloum

salloumnelly@gmail.com
Thurayya Arayssi

tha2002@qatar-med.cornell.edu

1 Saint-Joseph University, Hotel-Dieu de France Hospital, Beirut, Lebanon

2 Assiut University, Egypt and King Hamad University Hospital, Bahrain, Bahrain

3 King Hussein Medical Center, Amman, Jordan

4 Specialized Medical Center, Riyadh, Saudi Arabia

5 Cairo University, Cairo, Egypt

6 Dr Humeira Badsha Medical Center, Dubai, United Arab Emirates

7 Z-Clinic, Beirut, Lebanon

8 Jordan Hospital, Amman, Jordan

9 Weill Cornell Medicine, Doha, Qatar 